\title{
Implications of a Narrow Automated Vehicle-Exclusive Lane on Interstate 15 Express Lanes
}

\author{
Sahar Ghanipoor Machiani $\mathbb{D}^{1},{ }^{1}$ Alidad Ahmadi $\mathbb{D}^{2},{ }^{2}$ Walter Musial $\mathbb{D}^{2},{ }^{2}$ Anagha Katthe $\left(\mathbb{D},{ }^{1}\right.$ \\ Benjamin Melendez $\mathbb{D}^{1}{ }^{1}$ and Arash Jahangiri ${ }^{1}{ }^{1}$ \\ ${ }^{1}$ Department of Civil, Construction, and Environmental Engineering, San Diego State University, San Diego 92182, CA, USA \\ ${ }^{2}$ Linscott, Law \& Greenspan, Engineers, San Diego, CA, USA \\ Correspondence should be addressed to Arash Jahangiri; ajahangiri@sdsu.edu
}

Received 3 October 2020; Revised 14 December 2020; Accepted 5 January 2021; Published 18 January 2021

Academic Editor: N. N Sze

Copyright (C) 2021 Sahar Ghanipoor Machiani et al. This is an open access article distributed under the Creative Commons Attribution License, which permits unrestricted use, distribution, and reproduction in any medium, provided the original work is properly cited.

\begin{abstract}
The main objective of this study is to evaluate the safety and operational impacts of an innovative infrastructure solution for safe and efficient integration of Automated Vehicle (AV) as an emerging technology into an existing transportation system. Filling the gap in the limited research on the effect of AV technology on infrastructure standards, this study investigates implications of adding a narrow reversible AV-exclusive lane to the existing configuration of I-15 expressway in San Diego, resulting in a $9 \mathrm{ft} \mathrm{AV}$ reversible lane and, in both directions, two 12-feet lanes for HOV and FasTrak vehicles. Given the difference between the operation of AVs and human-driven vehicles and reliance of AVs on sensors as opposed to human capabilities, the question is should we provide narrower AV-exclusive roadways assuming AVs are more precise in lateral and longitudinal lane keeping behaviour? To accomplish the goal of the project, a historical crash data analysis and a traffic simulation analysis were conducted. Crash data analysis revealed that unsafe speed, improper turning, and unsafe lane change are the most recurring primary collision factors on I-15 ELs. AVs' automated longitudinal and lateral control systems could potentially reduce these types of collisions on an $\mathrm{AV}$-exclusive lane with proper infrastructure features for AV sensor operation (e.g., distinct lane marking). Microsimulation findings indicated an AV-exclusive lane may increase traffic flow and density by up to $14 \%$ and $24 \%$, respectively. It also showed that average speed is reduced. However, this could lead to the speed differential increase between the exclusive lane and adjacent lane requiring careful consideration if additional treatments or barriers are needed. The results of this study contribute to infrastructure adaptation to AV technology and future AV-exclusive lanes implementations.
\end{abstract}

\section{Introduction}

As the world finds itself at the beginning of the next industrial revolution, automated vehicles (AV) are one of the key drivers. The number of AVs on the roads is increasing with more and more people embracing the new technology. The National Highway Traffic Safety Administration (NHTSA) crash statistics data in 2018 revealed that the critical causes of vehicle crashes were driver-related errors (94\%), vehicle-related errors (2\%), environment-related factors $(2 \%)$, and other factors (2\%) [1]. Driver-related errors include recognition error, decision error, performance error, nonperformance error, etc. Vehicle-related errors include tires, brakes, steering, and engine errors, while environmental factors include slick roads, glare, view obstructions, signs and signals, and other weather-related factors. AVs are a viable option to greatly improve road safety by avoiding crashes that are typically driver induced. However, safety and performance of AVs in real-world conditions need to be carefully evaluated.

Safe deployment of AVs in real-world conditions requires modifications to the existing infrastructure. As today's AVs are in the early stages of autonomy, constant interaction of AVs with conventional vehicles might result in safety issues and traffic disruptions. Hence, introducing an $\mathrm{AV}$-exclusive lane as an infrastructure modification is a 
practical solution to minimize interactions between AVs and conventional vehicles.

The purpose of this study is to expand the knowledge base in terms of safety and operational impacts of exclusive freeway lanes for AVs, and to investigate implications of a narrow AV-exclusive reversible lane on I-15 in San Diego County, California, as a case study. The Interstate 15 (I-15) Express Lanes (ELs) Corridor, between State Route 163 (SR163) and Via Rancho Parkway, currently provide $4 \mathrm{HOV}$ and toll-paying FasTrak lanes divided by the moveable barrier. The lane combinations that can be provided, depending on peak direction and position of the moveable barrier that separates the northbound (NB) and southbound (SB) EL traffic, are $2 \mathrm{NB}$ and $2 \mathrm{SB}, 1 \mathrm{NB}$ and $3 \mathrm{SB}$, or $3 \mathrm{NB}$ and $1 \mathrm{SB}$ (see Figure 1(a)). Caltrans is seeking efficient ways to handle more traffic in the ELs, especially during rush hours or during major accidents when ELs are open to all traffic. In the available width between the fixed concrete barriers that separate the EL facility from the regular lanes, it would be possible to add a narrow reversible lane to be used only by AVs. This reversible AV lane for travel in the peak traffic direction would be $9 \mathrm{ft}$ wide and located next to the moveable barrier. In both the NB and SB directions of the EL, there would be two $12 \mathrm{ft}$ wide lanes for HOV and FasTrak vehicles and the outside shoulder next to the fixed barrier would be $8 \mathrm{ft}$ wide (see Figure $1(\mathrm{~b})$ ). With the new configuration, the question is what are the traffic implications and considerations of AV lanes and whether AVs could operate safely in a $9 \mathrm{ft}$ lane?

To accomplish the goal of the research, the following tasks were performed. First, a literature review was conducted related to AV lateral control technology, impacts of lane width, and impacts of AVs on the transportation system. Next, a detailed analysis of crash history of I-15 ELs was performed to understand the type and cause of crashes, at least, partially attributable to the AV system. Finally, a traffic microsimulation was carried out, modelling automation level 3-conditional automation, to understand the implications of having a narrow AV-exclusive reversible lane on I-15 ELs.

\section{Literature Review}

This literature review explores vehicle lateral control systems, lane width safety, AV safety, and the role of HD mapping in AV operations, establishing the state of science and background information related to this study. Understanding the limitations of current technology and the direction that research is going pertaining to AV operations must be considered as this study proceeds. Given that AVs operating on public roads is still in its nascent stages, there is a lack of available data and ample opportunity for future research to address the performance of AVs in the operational and safety contexts.

2.1. Lateral Control Systems. Lateral control technologies have developed iteratively, evolving from lane departure warning systems to lateral assist systems to lane centering systems. Lane Departure Warning (LDW) systems only warn the driver that their vehicle is departing from the intended lane. Amditis et al. [2] developed a lane departure avoidance system that is capable of handling varying traffic conditions. Through the use of environmental perception sensors such as cameras, radar, laser scanners, and GPS, input data are collected and perceived, a decision is formulated, and action is taken by the vehicle controller. Cualain et al. [3] present an LDW system with an image processing method utilizing multiple optical cameras. The authors found the proposed system to be more robust than single camera systems with higher detection rates. The proposed system used a lane segmentation strategy with a modified subtractive clustering algorithm. Zhang et al. [4] proposed an LDW system based on a camera supported analysis of grayscale distributions. An Advance Reduced Instruction Set Computing Machine- (ARM-) based platform was used to execute a lane departure risk evaluation model based on lasting time and frequency. Field tests yielded sufficient lane detection results.

Some research has been conducted to make LDW systems more accessible to a variety of consumers and vehicles. Hsiao et al. [5] created a handheld LDW system that can be mounted on vehicle dashboards. The algorithm developed uses a peak finding method with feature extraction that determines lane boundaries.

As LDW technology matured, research started to focus on the refinement of the systems, as well as making them more robust by coupling with other technology. Clanton et al. [6] explored coupling LDW with GPS technologies for enhanced LDW system accuracy. The controller system measured GPS error utilizing the LDW, enabling it to develop correction measures. In the event that the LDW system failed, using the precalculated correction measures, the GPS would assist in LDW functions until the LDW reestablished function. Enache et al. [7] proposed an active steering assistance system that acts as both a lane departure avoidance and a lane-keeping system. The authors' focus was on the lane-keeping performance of the steering assistance system while under the driver's control of the vehicle. The advancement of the LDW technology paved the way for more complex systems necessary for AV lateral control.

Lane-Keeping Assist (LKA) systems both warn and then assist the driver to return to the center of the lane if drifting is detected. The challenges for LKA are related to perceiving the environment and processing the information fast enough to aide in controller decision making. Wang et al. [8] explored the challenge of time delay associated with cameras' processing of imagery at different sampling rates impacting vehicle lateral control. The author presented a combined vision vehicle model to address the low sampling frequency and varying time delay of the geometrical modelbased state calculation method. Field tests of the proposed methodology showed that the system updated lateral position faster than current on-board measurement systems.

Zhao et al. [9] proposed a two-level vehicle lateral control system, where the upper level develops a desired steering angle based on perception information from vehicle sensors. A multimodel fuzzy control algorithm was designed 

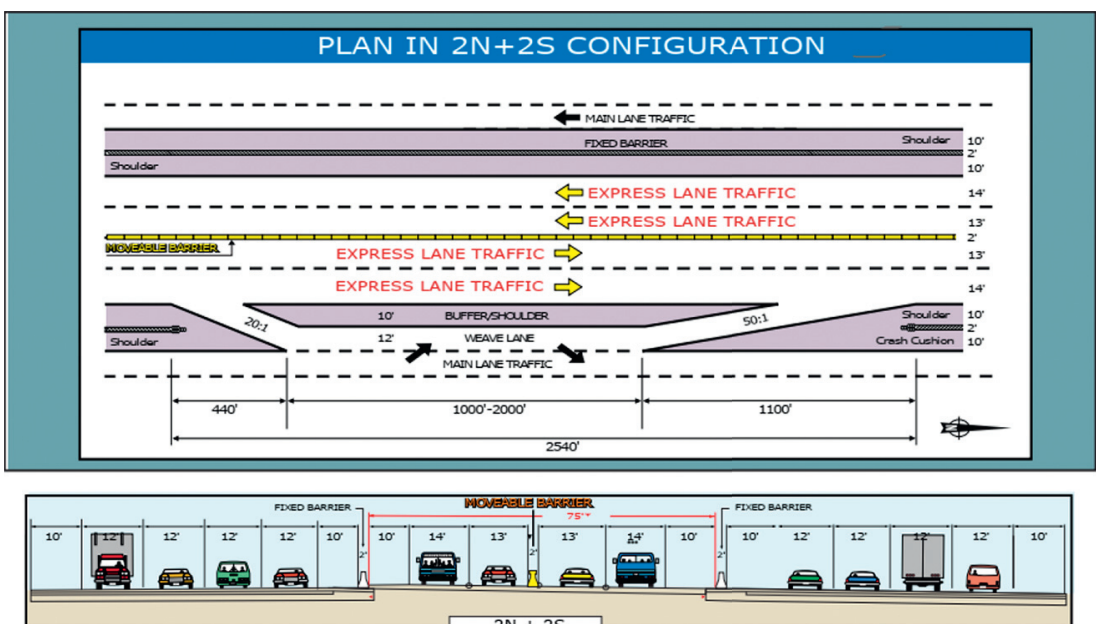

\begin{tabular}{|l|l}
$2 N+2 S$ \\
CONFIGURATION
\end{tabular}

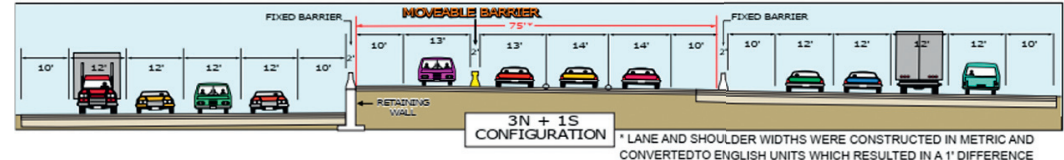

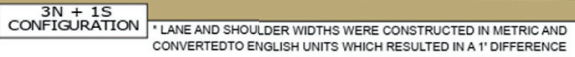

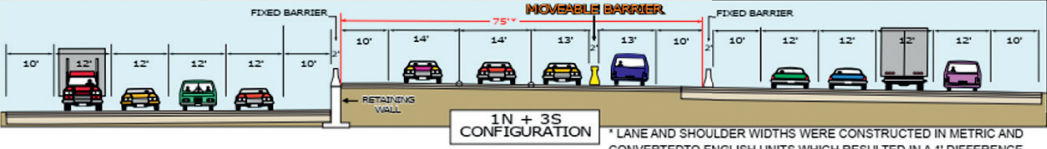

(a)
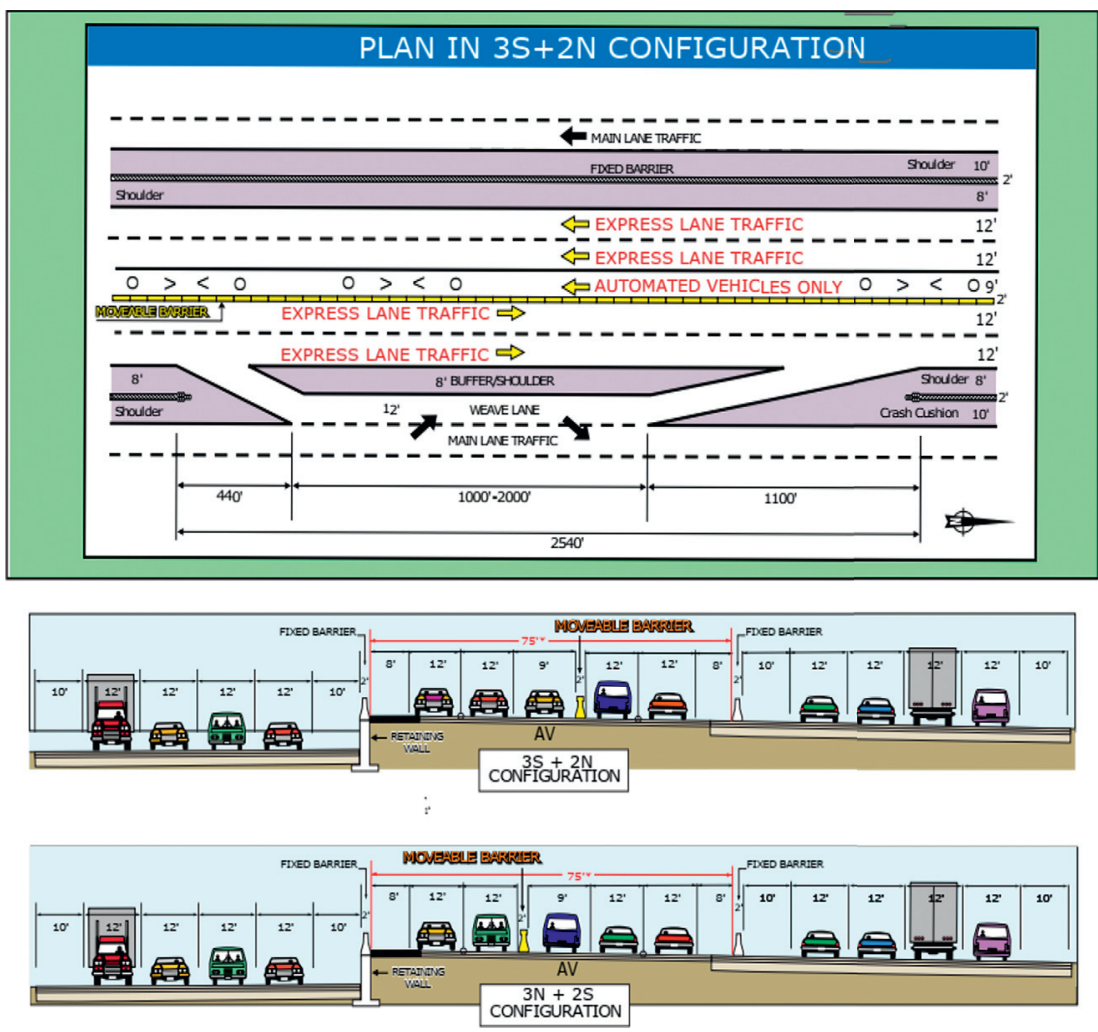

(b)

FIgURE 1: I-15 express lanes: (a) existing 4-lane configurations; (b) configurations with a reversible AV lane (plans courtesy of Caltrans). 
for lane tracking tasks in both the lane-keeping and lanechanging controllers. The lower level controller utilizes the calculated steering angle and generates the control signals for the steering actuators. As the LKA system matured, more focus was directed to their performance in all conditions. Mustaki et al. [10] propose an optimized lane-centering assist system (LCAS) (note that as the author describes LCAS, it is functionally an LKA system) that utilizes a multiscenario approach to consider performance when the system is affected by environmental factors (wind, curves, etc.), which was then tested in simulation.

As fully autonomous lateral control is the end state for AVs, the most recent research on lateral control focuses on Lane Centering (LC). Pendleton et al. [11] conducted an expansive literature review of current systems and algorithms pertaining to the operation of AVs. Of particular interest, the authors delved into detail the efficacy of various AV environmental perception systems such as LIDAR, cameras, INS/INU, and GPS. The authors also explore the various vehicle control strategies, with emphasis on geometric controls and model-based methods. Vehicle localization and the lack of updated topographic maps was identified as the overarching challenge to the system; however, the author notes advances in simultaneous localization and mapping (SLAM) that may address this.

Environmental perception is a key aspect of lane centering with ever increasing and more sophisticated research devoted to the topic. Ismail [12] discussed the design and implementation of the BlueBox computing system which enables the real-time perception capabilities of autonomous vehicles. Using various subsystems and sensors, the lanecentering assist system provides lane detection and tracking and is also capable of providing active steering to keep the vehicle automatically centered. The external environment is detected through forward-facing cameras and then steers to keep on track through lane detection and tracking algorithms. Berriel et al. [13] proposed a vision-based, real-time ego-lane analysis system that is capable of estimating egolane position, classifying lane marking types and road striping, performing lane departure warnings, and detecting lane changing events. The proposed system combines a number of environmental detection systems (cameras) using a single algorithm. Working in a temporal sequence, lane striping features are extracted from the cameras and a final estimated lane is calculated into a spline.

Broggi et al. [14] sought to address the challenge of designing a general-purpose path planner and an associated low-level control for autonomous vehicles operating in unknown environments. The model developed considered obstacle detection, ditch localization, lane detection, and global path planning. The vehicle environmental perception sensors helped generate a cost map which weighs obstacles and helps determine the traversable areas. To address the time delay associated with processing the perception data, way point coordinates were established for the drivetrain to follow, considering vehicle dynamics and path tracking information. The model exhibited a mean cross track error of $0.13 \mathrm{~m}$ in autonomous tests and $0.17 \mathrm{~m}$ in the leader follower mode.
These lateral control systems are predominantly vision based, with capabilities beyond just lane detection, notably obstacle detection. The ability to detect obstacles is essential for AVs to avoid debris in the roadway, as well as to aid in avoidance of side swiping collisions. To address the reality of dynamic driving conditions, some researchers have sought to make controllers more responsive. Lee and Litkouhi [15] discussed an automated lane=centering and -changing control algorithm that focused on enhancing the control accuracy of the vehicle. The proposed algorithm is capable of providing smooth and aggressive lane centering/changing manoeuvres according to current traffic conditions and driver preferences. The generated path could be recalculated for smoother or more aggressive lateral motion control.

Lateral control research has also considered the role of the vehicle's drivetrain and handling characteristics, particularly when it pertains to active steering to maintain the vehicle in the center of the lane. Most research previously simplified the vehicle model to act as a bicycle, meaning each axle was modeled as one wheel. Chebley et al. [16] presented a coupled control algorithm for longitudinal and lateral dynamics of an AV. Unlike most models which simplified vehicles to the bicycle model, their algorithm considered all parts of the vehicle and their interconnectedness. The algorithm used Lyapunov functions to ensure robust tracking of the reference trajectory/path in lane-changing actions, as well as obstacle avoidance and lane keeping. The objective of minimizing lateral displacement error whilst maintaining a desired longitudinal speed was achieved by generating a steering angle and a driving/braking torque that enable successful tracking of the reference trajectory. Attia et al. [17] posited an automated steering strategy based on nonlinear model predictive control. This strategy simultaneously considered the power train dynamics to manage the longitudinal speed tracking challenge in order to improve combined control. The prediction model calculates the future states of the dynamic system on a fixed finite time horizon. Tested in simulation against a predefined GIS trajectory, the lateral position error of the vehicle never exceeded $6 \mathrm{~cm}$, whilst heading angles are admissible and longitudinal speed is correctly tracked.

Xu et al. [18] and Filho et al. [19] were concerned with maintaining fidelity of the desired track with the predicted track of the lane centering system. Xu et al. addressed lateral control by developing a sliding mode control to manage vehicle dynamics at high speeds. The drive control system used a parameterized cubic spline interpolation function to calculate a desired vehicle trajectory. In field tests, the system exhibited a max lateral position error of $0.5 \mathrm{~m}$, with most error below $0.2 \mathrm{~m}$ when compared against a predetermined GPS trajectory. Filho et al. proposed a simplified control system for AVs that relied on a reduced number of parameters that could be set. To address lateral control, a cubic Bezier curve is utilized to correct the trajectory between the origin of the vehicle and the desired path. During field testing, approximately zero mean cross track error and an orientation error of -1.0397 degrees to 0.9225 degrees were observed. 
The direction and state of research and science of $\mathrm{AV}$ lateral control is important to ascertain for this study because it is imperative to understand vehicle capabilities and limitations when operating in a space-constrained environment. Given the reduced lane sizes and potential vehicle lateral separation, AVs must be able to maintain a course without deviation lest unsafe situations develop with potential catastrophic consequences.

\subsection{Lane Width Safety and Functional Impacts. Public} agencies are very sensitive to the impact of roadway infrastructure modifications. These impacts are quantified in crash modification factors [20]. Gross et al. [21] researched the impact of shoulder/widths combinations on crash rates and developed corresponding safety performance factors. The authors found that reallocating lane and shoulder widths given a fixed total pavement width can be a cost-effective measure for reducing crashes on rural, two-lane undivided highways. Gross et al. posit that, for narrow widths, slight reductions in crashes can be achieved by adding shoulder widths compared to lane widths, but only in low traffic scenarios. Lee et al. [22] developed a comprehensive safety model with safety performance factors. The authors found that, in general, shoulder widths have a more substantial impact on safety when the lane width is narrow. The study also indicated that crash modification factors increase with decreasing lane or shoulder width. Labi et al. [23] provide an in-depth discussion on the lane width and shoulder width relationship with crashes and costs and present decision support charts that can be used by highway agencies to determine the optimal lane and shoulder widths.

It is important to also consider the functional impacts of lane width reduction, especially in regards to flow, speed, and level of service. An FHWA research initiative [24] cited that the 1985 HCM found that a roadway with 9foot lanes and no shoulders could only support $2 / 3 \mathrm{ca}-$ pacity of a two-lane roadway with 12 -foot lanes and 6-foot shoulders. The report found no flow benefits in reducing lanes to 10 feet or 9 feet. Rosey et al. [25] compared simulator-derived data to a previous field study regarding the impact of lane width reduction on speed. The researchers found that simulator results corroborated previous field studies on speeds. They found that speeds remained unaffected by lane narrowing; however, drivers tended to move towards the centerline after narrowing and moving to the right (outside edge of lane) prior to meeting an oncoming vehicle. Dorothy and Thieken [26] explored the relationship between a number of different highway design variables such as speed, level of service, physical characteristics of the design vehicle, and capabilities of the driver. In reference to lane width, the authors consider the recommendations of the Greenbook of 2004, "9-foot lanes are appropriate on low-volume roads in rural and residential areas, or in urban areas, inside lane to accommodate wider shared use outside lanes." It is important to note that these aforementioned considerations are all heavily linked to human factors and may be null and void in dedicated AV lane scenarios.
Given that the proposed AV lanes will be reduced to nine feet, it is worth examining prior research into the operational impacts of reduced lane widths. While this research pertains to AVs, regular non-AV cars will be in operation for many years to come. Drivers will have to interface with AVs in these shared roadway environments, inducing some of the impacts and considerations described in this review.

2.3. AVs Safety and Functional Impacts. Focusing on safety, Giuffre et al. [27] consider the benefits and costs associated with AV technology in context of safety improvements on highways. The authors posit that autonomous vehicles have the potential to reduce time headway, thus enhancing traffic capacity, and improve safety margins in car following. They also identified crash safety factors such as cyber attacks, systems failures, and database deficiency that must be considered. Finally, the authors conducted a microsimulation of mixed conventional and autonomous vehicles. New autonomous vehicle-centric accident modification factors are recommended.

The Victoria Transport Policy Institute [28] created a report that examined the major risks, benefits, and planning consideration for autonomous vehicles as they deploy onto public rights of way. Potential risks identified include: hardware and software failures, malicious hacking, and platooning risks (i.e., increased crash severity due to higher vehicular densities and risks associated with human drivers entering platoons). The report cites/recommends that, for platooning of $\mathrm{AVs}$ to be safe and effective, dedicated AV lanes may be required. The European Road Assessment Programme [29] developed a comparison between how AVs and human operated vehicles behave and react in various safety-related scenarios. Various influencers in AV crash configuration/scenarios are considered, as well as corresponding infrastructure attributes. The authors advocate the need for clear and consistent signage that is well maintained, as well as for clear and robust striping. Additionally, investments in connectivity of infrastructure are also stressed.

Finally, the functional impacts of AV deployment are also beginning to be considered. Hamilton et al. [30] focused on identifying and evaluating opportunities, constraints, and guiding principles for implementing AV lanes. Utilizing a simulator-based model, the researchers identified parameters and variables that were sensitive to dedicating lanes to $\mathrm{AV}$ users and identified expected impacts under various conditions. Lanes were delineated based on AV market penetration rates (i.e., percentage of vehicles in the traffic mix with AV capabilities/AV attributes) by using "lane friction," speed differential between the dedicated lanes and adjacent general-purpose lanes, as a safety measure. The authors posit that AVs will benefit most from dedicated lanes (DL) when AV market penetration is low. Recommendations include (1) shared DL with HOVs at lower market penetration rates, (2) exclusive DLs at medium market penetration (20-45\%), and (3) no DLs for higher market penetration.

Using computer simulation, Ye et al. [31] examined traffic flow throughput on various dedicated AV lane 
configurations on a three-lane highway. The researchers found that it is most beneficial for traffic flow throughput with one CAV DL when CAV market penetration rate exceeds $40 \%$ and two CAV DLs when CAV market penetration exceeds $60 \%$. It was also discovered that, at lower market penetration rates, CAV DLs had a negative impact on the overall throughput, yet at very high CAV penetration rates, positive effects on flow and density also decrease.

As AVs are still new to the roadway system, there is a dearth of data to measure and analyse their functional and safety impacts on the transportation system. There are obvious limitations to predictive models based on simulations. Undoubtedly, as AVs become more prevalent, the availability and quality of the input data for future studies will improve, resulting in improved research outcomes.

2.4. AVs and High-Definition Mapping. GPS locational software and high-definition (HD) maps are integral to many AV development programs and research; Seif and $\mathrm{Hu}$ [32] explore the state of science and research, context, and implication of HD maps in assisting autonomous vehicle navigation. Bauer et al. [33] demonstrate the benefits of integrating HD maps, GPS location data, and vehicular odometer data through a particle filter-based localization algorithm. Liu et al. [34] explore the potential capabilities of HD maps in AV operation, analysing HD map-based vehicular localization. Zheng and Wang [35] develop a localization system utilizing HD maps as sensors, while also exploring the influence of geometry as a factor affecting locational accuracy. Kuhn et al. [36] argue that vehicles require detailed prior knowledge (in the form of HD maps) of the planned route before the beginning of a journey. Vehicle localization can occur faster and more accurately, as the car navigation system compares maps and sensor data derived from its surroundings. Whether HD maps are a primary or back up means of navigation is yet to be seen, and it will likely play an important role in AV development.

\section{Methodology}

3.1. Investigation of I-15 Express Lanes Crash History. Crash data provide important information, such as type, severity, and potential cause of crash, and could illuminate potential shortcomings of operating AVs on the I-15. The descriptive study approach was employed to observe leading factors in accidents on this particular portion of the I- 15 . These factors could serve as the basis for future research into how these factors could be mitigated by AVs (available realworld data dependent). The crash study also points to key design specifications (e.g., distinct lane markings) that need to be considered while designing the dedicated AV lane as it relates to safe operation of AVs. Historical crash data on the I-15 ELs were examined. The primary source of data for conventional accident information was the California Highway Patrol's Statewide Integrated Traffic Records System (SWITRS) database.

Data were selected based on location, jurisdiction, and year. Ten years (2009-2018) of data were collected for the
I-15 corridor which included three csv files: collision data, party data, and victim data. The three files were combined based on the same crash event number in each of these files. Each crash event number was sometimes observed to have multiple vehicles or injuries/fatalities associated with it. In this study, all the vehicles involved in a particular crash event were considered in the analysis. Roadway shapefiles from Caltrans were used to filter only those data points (i.e., crash locations) that were in the designated area of interest (I-15 ELs from SR 52 to SR 78). The filtered data points with their associated attributes were exported and used for further analysis. A total of 717 crash events were observed from 2009-2018 on the study site. When considering all vehicles involved in each crash event, 1473 crashes were analysed. Some of the attributes considered in this study are as follows: primary collision factor, type of collision, and collision severity. Figure 2 shows crashes datapoints for the study area (SR 52 to SR 78). Each blue dot on the map represents a crash. A part of the ELs is represented in a magnified window where crashes are clearly visible.

\subsection{Impact Analysis Using Microsimulation.} Microsimulation was used to evaluate the impact of implementing the proposed exclusive AV lane on the Interstate (I-15) ELs. To best understand the transportation effect of the project, a sensitivity analysis was conducted for three scenarios as noted below. The microsimulation model was developed with the Caliper TransModeler SE version 5.0 software package. The following sections will discuss, in more detail, the microsimulation input assumptions and output metrics used for the evaluation.

Scenario 1- EX: the baseline/calibration scenario with existing volumes/network

Scenario 2- AV: existing volumes/network with AV adoption

Scenario 3- AVL: existing volumes with the proposed $\mathrm{AV}$-exclusive lane and adoption

The simulation investigates both safety and operational aspects examining metrics such as average speed (safety and operational measure), speed differential (safety measure), average density (operational measure), and flow (operational measure).

3.2.1. Corridor Network. The microsimulation evaluates a section of the I-15 ELs corridor, approximately 7 miles in length, between Ted Williams Freeway (State Route 56) to State Route 163. The ELs were modeled in the simulation environment including all physical features such as merging and diverging points, acceleration/deceleration lanes, Direct Access Ramps (DAR), lane/shoulder configurations, and width. Figures 3(a) and 3(b) illustrate the extent and configuration of the network. The network was divided into 12 segments as numbered on the figure. The microsimulation outputs are collected in the middle of each segment on an individual lane basis. 


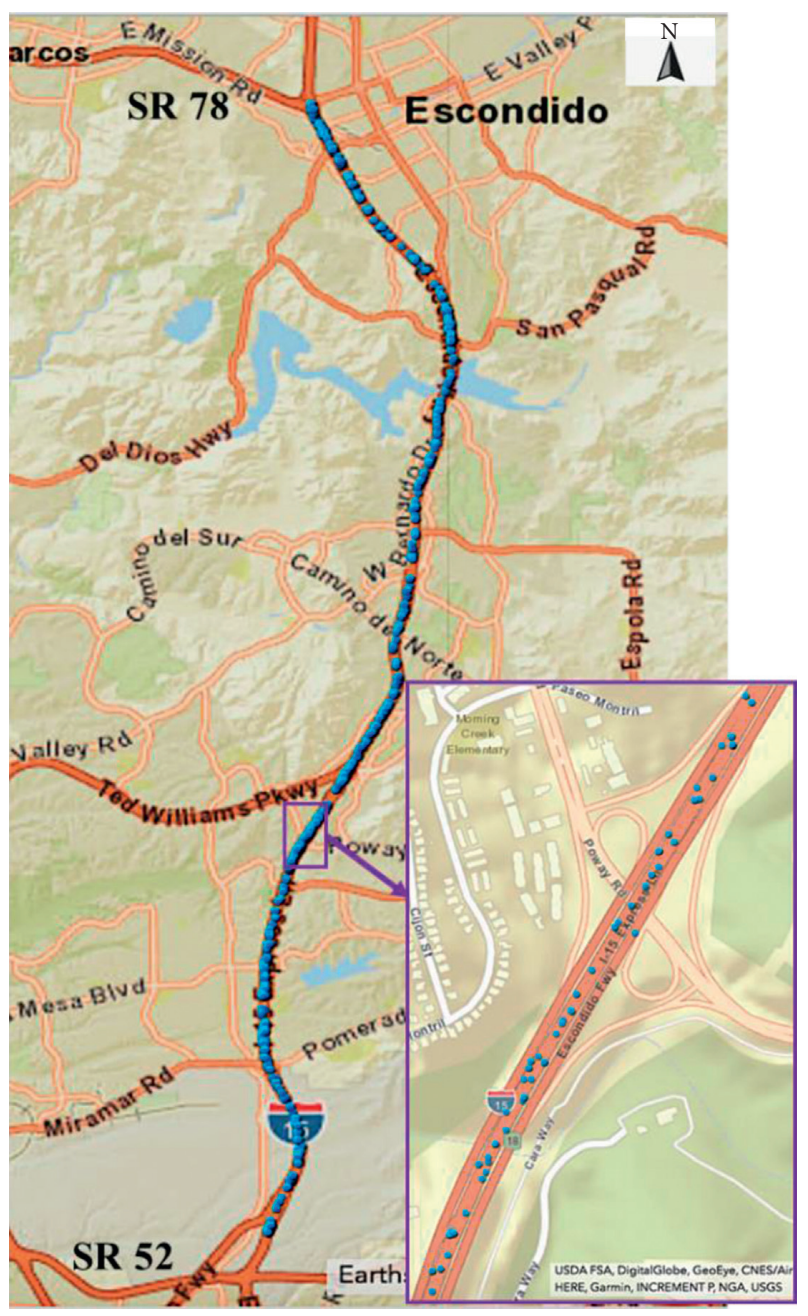

Figure 2: Crashes (2009-2018) on I-15 express lanes from SR-52 to SR-78.

Figure 3(c) illustrates the lane configuration under scenario 3 for the southbound direction during the AM peak hour. The AM peak hour scenario was selected since it is the most conservative period given a more critical traffic condition occurs during AM in comparison to PM peak hour.

3.2.2. Input Assumptions. In addition to physical features of the network, microsimulation parameters were modified to reflect the field conditions. Heavy vehicles were not modeled assuming they are not permitted on the ELs. Non-AVs were evaluated with the Modified General Motors Car-Following Model which is the default setting of the software. Per software developer's guidance, AVs were evaluated with the Constant Time Gap Car-Following Model.

Under scenarios 2 and 3, AVs were modeled with automation level 3-conditional automation. Level 1 and 2 automations were not considered given they represent driver support rather than true vehicle automation. Under scenarios 1 and 2, AVs were assumed to have the same deviation from the speed limit as non-AVs. This assumes that approximately $30 \%$ of drivers are traveling within the speed limit. Under scenario 3, level 3 AVs are not affected by non-
AVs as they are traveling on the exclusive lane. Therefore, the AVs are assumed to travel at the speed limit.

The AV Market Penetration Rate (MPR) for the baseline scenario was assumed zero since level $3 \mathrm{AV}$ vehicles are currently not available. Scenarios 2 and 3 assumed varying level 3 MPRs of $15 \%, 30 \%$, and $45 \%$. This approach provided sensitivity to the analysis given its difficulty to predict MPR with certainty.

Each microsimulation was run for 60 minutes using the peak-hour volumes. A maximum warmup period of 10 minutes was also assumed to preload the network.

3.2.3. Baseline Volumes. Baseline volumes and speeds were received from Caltrans. The extracted data represent morning peak-hour (7AM-8AM) volumes and speeds on HOV lanes from Tuesday October 15, 2019, to Thursday October 17, 2019.

3.2.4. Calibration. Existing field volumes were input into the software to verify that the microsimulation accurately represents field conditions. Volumes, in veh/hr, and speeds, in $\mathrm{mph}$, yielded from the model were then compared to available field data. In addition, the Geoffery E. Havers (GEH) value was used for the calibration process and to assess how the microsimulation outputs match the field conditions. A Low GEH, under five, indicates a well-calibrated model. The average GEH of 0.40 and 0.77 was achieved for the northbound and southbound directions, respectively (see Table 1).

\section{Results and Discussion}

4.1. Investigation of I-15 Express Lanes Crash History. The I-15 ELs crash dataset contains a number of different violation categories referred to as primary collision factors (PCF) indicating the main reason for a crash (Figure 4(a)). To further understand the specifics of a crash, the type of collision and collision severity were analysed in conjunction with the PCF (Figures 4(a)-4(c)). In the PCF graph, more than half of all crashes were due to unsafe speed (55\%, count: 814 crashes), 19\% (count: 279 crashes) were due to improper turning, and 13\% (count: 196 crashes) were due to unsafe lane change. From the type of collision graph, it was observed that rear-end collision dominated the list with 55\% (814 crashes) of all crashes recorded, followed by sideswipe (22\% each, 317 crashes) and hit-object (16\%, 234 crashes). In the collision severity graph, although there were very few cases of fatality $(0.5 \%, 8$ fatalities), many human injuries were reported ranging from complaint of pain to severe injury (36\%). Property damages accounted for $36 \%$ of the crash consequences leaving behind $27 \%$ of no injury cases. It should be noted that, in this study, every vehicle involved in the crash event is mapped to the highest degree of collision severity experienced by any of the passengers.

The three main PCFs were further analysed in combination with the type of collision and collision severity to sketch the cause, effect, and consequence relationship (see Figure 5). 


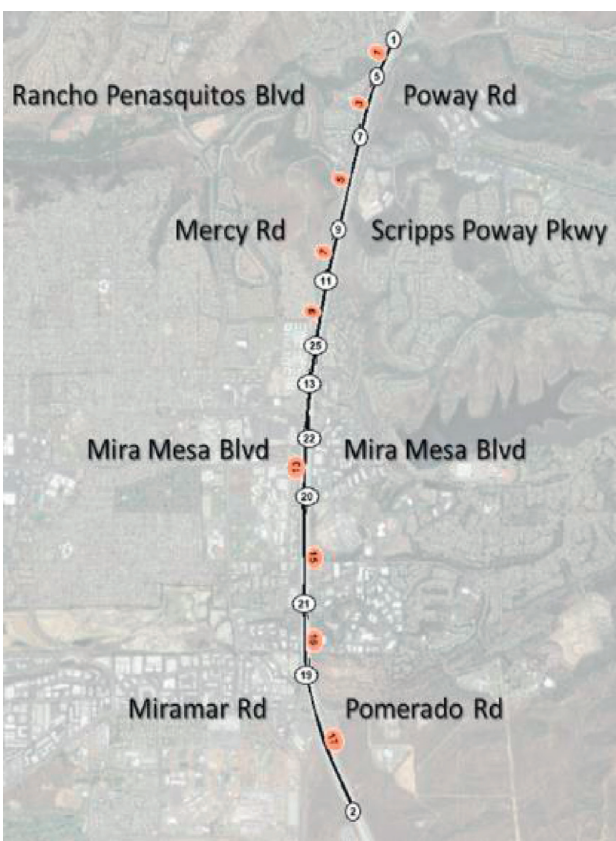

(a)

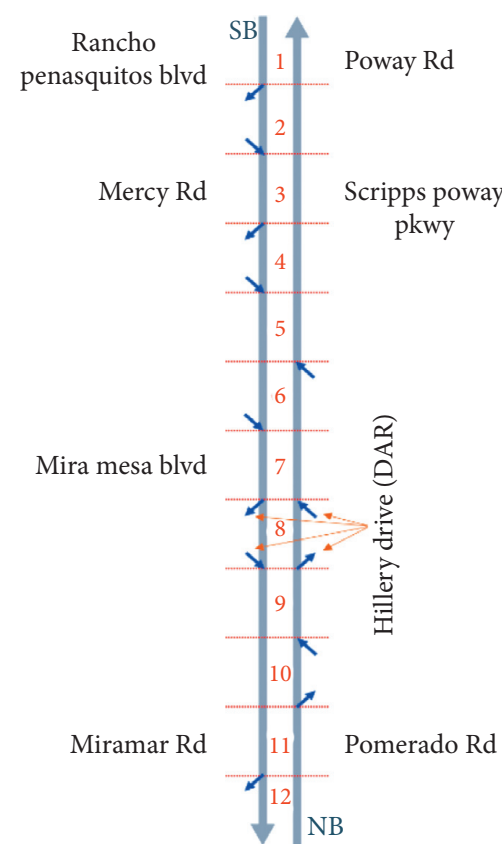

(b)

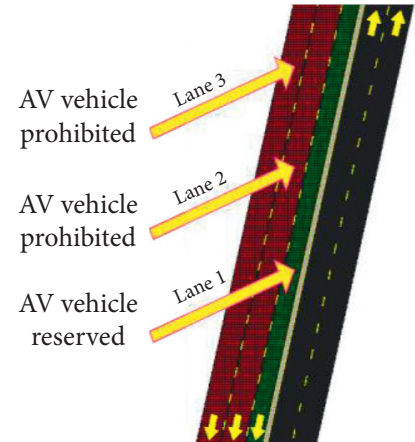

(c)

Figure 3: (a) Network extents, (b) network features, and (c) a sample of the road in scenario 3.

TABle 1: Simulation calibration.

\begin{tabular}{|c|c|c|c|c|c|c|c|c|c|}
\hline \multirow[b]{2}{*}{$\#$} & \multirow[b]{2}{*}{ Location } & \multirow[b]{2}{*}{ Dir } & \multirow{2}{*}{$\begin{array}{l}\text { Field data } \\
\text { Volume }\end{array}$} & \multicolumn{2}{|c|}{ Simulation output } & \multirow{2}{*}{$\begin{array}{l}\Delta \text { volume } \\
(\%)\end{array}$} & \multirow{2}{*}{$\begin{array}{c}\Delta \text { average speed } \\
(\%)\end{array}$} & \multirow[b]{2}{*}{ GEH } & \multirow[b]{2}{*}{ Average speed } \\
\hline & & & & $\begin{array}{l}\text { Average } \\
\text { speed }\end{array}$ & Volume & & & & \\
\hline \multirow{2}{*}{1} & Rancho Penasquitos Blvd/Poway & NB & 800 & 71.3 & 775 & 71.7 & -3 & 1 & 0.89 \\
\hline & $\mathrm{Rd}$ & SB & 3500 & 75.9 & 3499 & 72.5 & 0 & -4 & 0.02 \\
\hline \multirow{2}{*}{2} & \multirow{2}{*}{ Mercy Rd/Scripps Poway Pkwy } & NB & 700 & 66.2 & 689 & 70.7 & -2 & 7 & 0.41 \\
\hline & & SB & 2500 & 75.5 & 2474 & 70.5 & -1 & -7 & 0.52 \\
\hline \multirow{2}{*}{3} & \multirow{2}{*}{ Mira Mesa Blvd } & NB & 800 & 69.0 & 792 & 73.0 & -1 & 6 & 0.29 \\
\hline & & SB & 3400 & 71.9 & 3340 & 70.3 & -2 & -2 & 1.04 \\
\hline \multirow{3}{*}{4} & \multirow{2}{*}{ Miramar Rd/Pomerado Rd } & NB & 800 & 73.6 & 800 & 76.5 & 0 & 4 & 0.00 \\
\hline & & SB & 3000 & 73.7 & 2917 & 70.2 & -3 & -5 & 1.52 \\
\hline & \multicolumn{5}{|c|}{$\begin{array}{c}\text { Average NB } \\
\text { Average SB }\end{array}$} & & $\begin{array}{l}-1.4 \\
-1.4\end{array}$ & $\begin{array}{c}4.3 \\
-4.6\end{array}$ & \\
\hline
\end{tabular}

${ }^{*}$ Field volumes are rounded to nearest hundred.

Unsafe speed is the most important PCF that contributed to around $55 \%$ of the total crashes. It can be observed that most crashes involved rear-end collisions that accounted for $87.1 \%$ of total unsafe speed crashes (count: 709 crashes), among which human injuries, ranging from complaint of pain to severe injury, and property damage were estimated to be $33 \%$ and $34 \%$, respectively. However, no fatalities were observed. The second and third highest categories are hit object (6.1\% of total unsafe speed crashes, count: 50 crashes) and sideswipe (4.2\% of total unsafe speed crashes, count: 34 crashes) collisions. Of the total 3 fatalities, hit object and sideswipe collisions accounted for one fatality each.

Crashes caused due to unsafe speed can potentially be reduced with the use of AVs, as they are supposed to perfectly comply with speed limits. AVs follow good speed discipline with less variability and maintain close to accurate bumper to bumper spacing, provided the performance of environmental sensors are accurate and reliable. Even if one or more sensors failed, there should be sufficient redundancies in the system to mitigate performance degradation significantly.

The next PCF analysed was improper turning that contributed to around $19 \%$ of total crashes on I-15 ELs. Hit object collisions (41.9\% of total improper turning crashes, count: 117 crashes) in this category mostly resulted in property damage and human injuries ranging from complaint of pain to severe injury. It also resulted in one fatality. The second highest collision type was sideswipe collisions (38.3\% of total improper turning crashes, count: 107 crashes) with collision severity ranging from complaint of pain to severe human injuries and property damage. Besides hitobject and sideswipe, a small number of rear-end ( $8 \%$ of total 


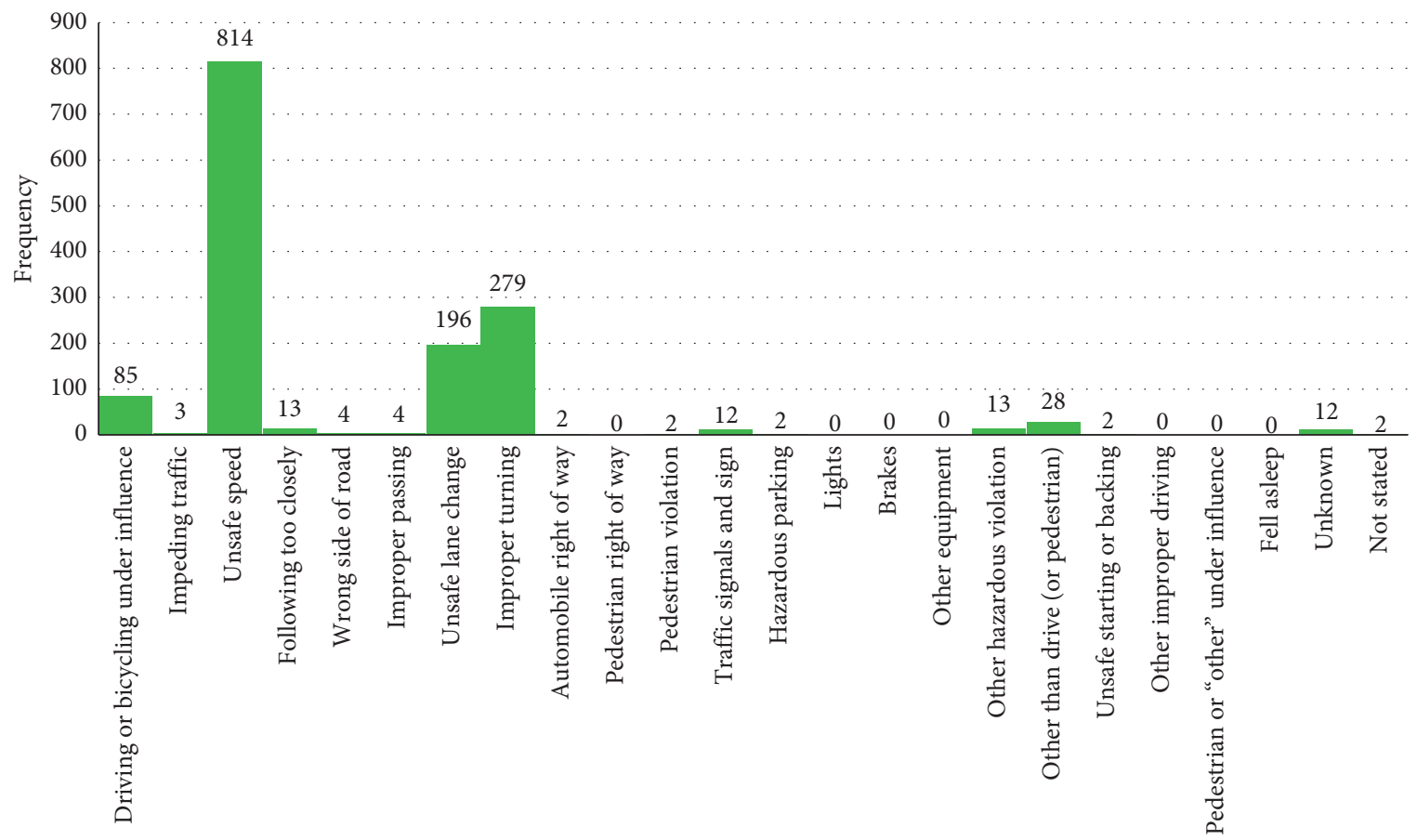

(a)

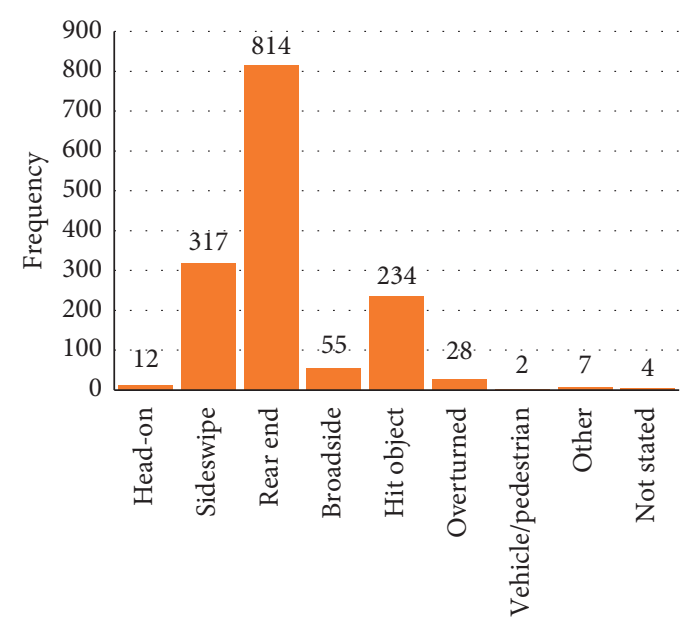

(b)

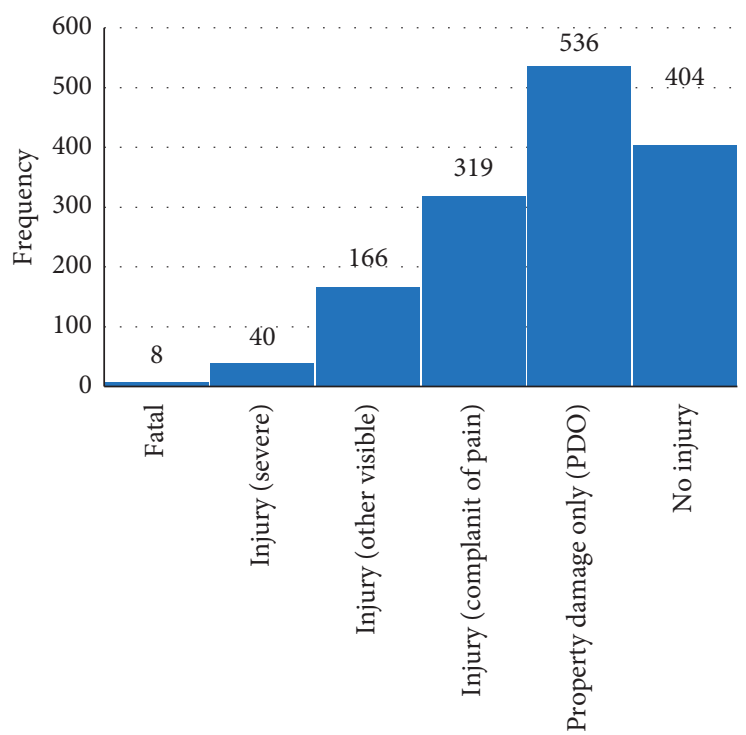

(c)

FIgure 4: (a) Primary collision factor (PCF), (b) collision type, and (c) collision severity. 


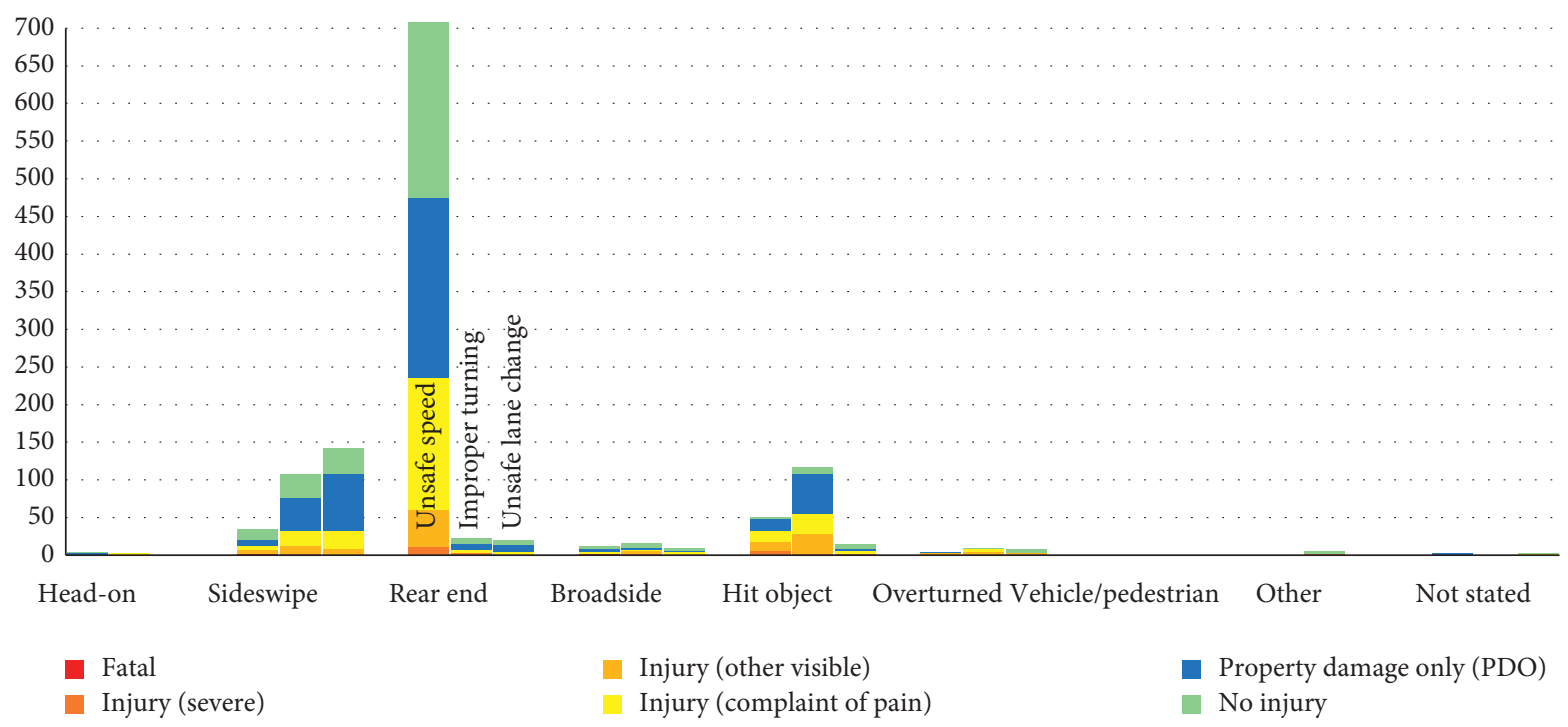

Figure 5: Types of collisions for different collision severities attributed to 3 main PCFs.

improper turning crashes, count: 22 crashes), overturned (4\% of total improper turning crashes, count: 10 crashes), and broadside collisions (6\% of total improper turning crashes, count: 16 crashes) were observed.

AV attributes such as lane keep assist (LKA) systems have the capability to prevent the vehicle from drifting from its desired path, thus avoiding improper turning. Additionally, lane infrastructure needs to be designed carefully, such that the barrier/median is detected and interpreted by the AV sensors correctly with little room for error. Welldesigned and functioning environmental sensors, signboards, and markings are required for safe travel of AVs on AV-exclusive lane. However, AVs are susceptible to turning errors when the weather conditions are adverse or if the appropriate sensors fail; hence, caution should be exercised when designing the $\mathrm{AV}$ and the $\mathrm{AV}$-exclusive lane to promote proper turning at all times.

The next important PCF observed on I-15 EL was unsafe lane changes that accounted for $13 \%$ of total crashes on I-15 ELs. Crashes due to unsafe lane changes resulted mainly in sideswipe collisions (72\% of total unsafe lane change crashes, count: 142 crashes) causing property damage and human injuries ranging from complaint of pain to visible injury. A few unsafe lane change crashes resulted in rear-end and broadside collisions (10\% and $5 \%$ of total unsafe lane change crashes, count: 20 and 10 crashes, respectively) that caused property damage and human injuries ranging from complaint of pain to visible injury. Hit object and overturned collisions caused property damage and human injuries ranging from complaint of pain to severe injury. No fatality crashes were observed during unsafe lane change crashes.

AV attributes such as lane departure warning systems (LDW), LKA systems, and lane centering can help to reduce unsafe lane departures resulting in safe commute on ELs. As mentioned, caution should be exercised when designing $\mathrm{AVs}$ and AV-exclusive lanes to avoid unsafe lane changes due to adverse weather conditions and/or sensor failure. Restricted access to an AV-exclusive lane from GPL can also prevent unsafe lane changes. The points of access from/to the AV-exclusive lane to/from GPL need to be carefully designed and monitored. Considering proper infrastructure design at these access points will prevent crashes due to unsafe lane departures, as well as improper turning.

4.2. Impacts Analysis Using Microsimulation. The microsimulation results were reviewed under four selected metrics including traffic flow, average density, average speed, and speed differential.

4.2.1. Traffic Flow. Traffic flow, in veh/hr, for all lanes was collected on each segment of the network. Table 2 shows, in more detail, percent change in the flow of traffic on each segment compared to scenario 1 (baseline scenario). Under scenario 2, the introduction of level $3 \mathrm{AVs}$ into the existing network does not show any measurable change in traffic flow. Under scenario 3, however, the introduction of level 3 AVs on an AV-exclusive lane resulted in up to $14 \%$ increase in traffic flow depending on the corridor location and AV MPR.

4.2.2. Average Density. Average density is calculated for the length of the segment in vehicles per mile per lane. Figure 6 demonstrates average density along the corridor. Similar to traffic flow, changes in average density were insignificant in scenario 2 . The average density of most segments was found to increase by up to $24 \%$ under scenario 3 .

It should also be noted that the $45 \%$ MPR scenario observed a significant increase in density on segment 10 . It is suspected that this is related to the compound effect of an existing curve, high ramp volumes, and higher AV-exclusive lane saturation.

4.2.3. Average Speed. The average speed of each segment was also evaluated (Figure 6). Consistent with the traffic flow and density, under scenario 2, no measurable difference was 
TABle 2: Traffic flow change from EX.

\begin{tabular}{|c|c|c|c|c|c|c|}
\hline \multirow{2}{*}{ Segment ID } & \multicolumn{3}{|c|}{ Scenario 2} & \multicolumn{3}{|c|}{ Scenario 3} \\
\hline & AV $15 \%$ & AV 30\% & AV $45 \%$ & AVL $15 \%$ & AVL $30 \%$ & AVL $45 \%$ \\
\hline 1 & $0.0 \%$ & $0.0 \%$ & $0.0 \%$ & $0.0 \%$ & $0.1 \%$ & $0.0 \%$ \\
\hline 2 & $0.1 \%$ & $0.1 \%$ & $0.1 \%$ & $0.0 \%$ & $0.0 \%$ & $-0.1 \%$ \\
\hline 3 & $0.0 \%$ & $0.1 \%$ & $0.1 \%$ & $0.0 \%$ & $-0.1 \%$ & $-0.2 \%$ \\
\hline 4 & $-0.3 \%$ & $0.1 \%$ & $0.0 \%$ & $3.0 \%$ & $7.2 \%$ & $10.7 \%$ \\
\hline 5 & $-0.1 \%$ & $0.0 \%$ & $0.0 \%$ & $2.7 \%$ & $6.9 \%$ & $10.2 \%$ \\
\hline 6 & $-0.2 \%$ & $-0.4 \%$ & $-0.2 \%$ & $2.8 \%$ & $6.6 \%$ & $10.0 \%$ \\
\hline 7 & $0.0 \%$ & $-0.1 \%$ & $0.2 \%$ & $2.7 \%$ & $5.9 \%$ & $9.1 \%$ \\
\hline 8 & $-0.2 \%$ & $0.2 \%$ & $0.0 \%$ & $3.0 \%$ & $6.3 \%$ & $9.9 \%$ \\
\hline 9 & $0.0 \%$ & $0.4 \%$ & $0.2 \%$ & $2.8 \%$ & $5.4 \%$ & $8.4 \%$ \\
\hline 10 & $-0.4 \%$ & $0.3 \%$ & $-0.2 \%$ & $2.1 \%$ & $5.1 \%$ & $8.3 \%$ \\
\hline 11 & $-0.3 \%$ & $0.0 \%$ & $0.2 \%$ & $2.2 \%$ & $5.1 \%$ & $7.7 \%$ \\
\hline 12 & $0.0 \%$ & $0.1 \%$ & $-0.2 \%$ & $4.4 \%$ & $8.7 \%$ & $13.6 \%$ \\
\hline
\end{tabular}

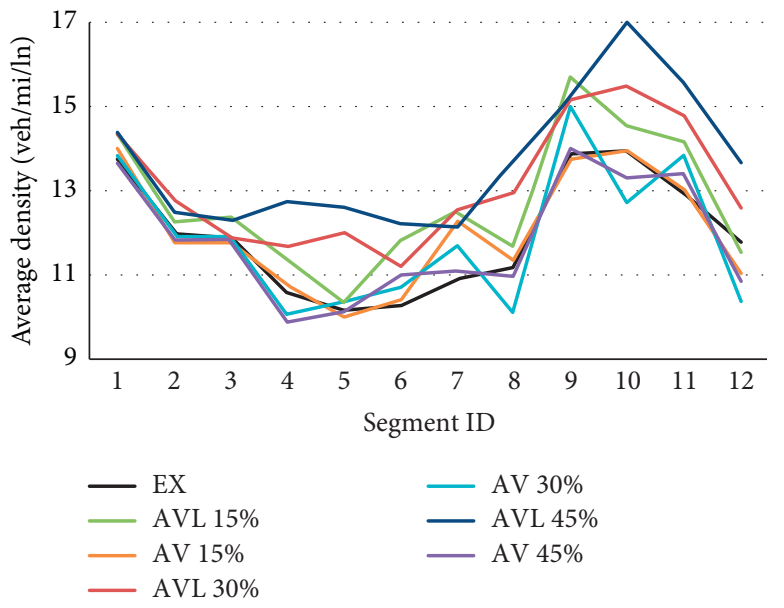

(a)

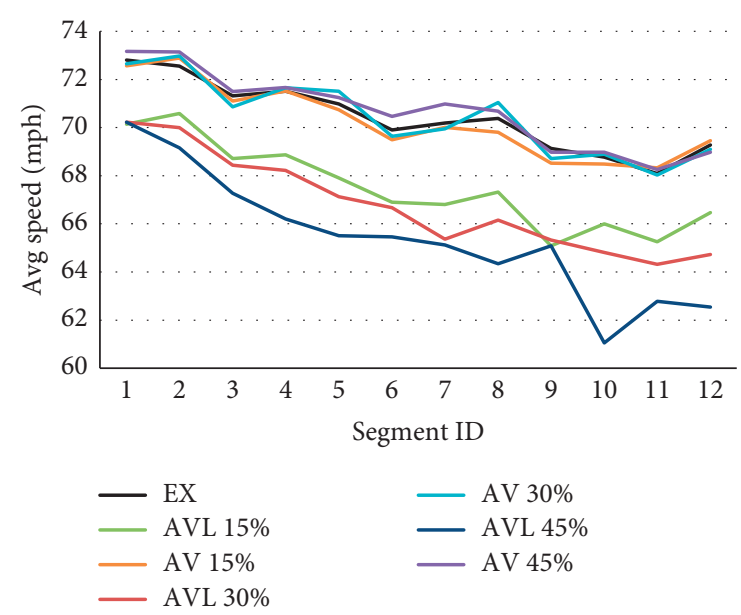

(b)

FIgURE 6: Average density and average speeds.

observed on average speeds. Under scenario 3, the average speed declined by $2-8 \mathrm{mph}$ depending on the location and AV MPR. The drop in speed was expected given the AVs were assumed to travel at the speed limit in the model. As indicated in the "input assumptions" section, this compares to only $30 \%$ of non-AVs and the AVs in scenario 2, which were assumed to travel at the speed limit.

4.2.4. Speed Differential. The speed differential between the lanes 1 (adjacent to the left shoulder) and 2 were determined on each segment. It is important to note that, under scenario 2, lanes 1 and 2 have similar characteristics (i.e., ELs). Scenario 3 introduces a distinction between lane 1 (i.e., AVexclusive lane) and lane 2 (i.e., EL). Table 3 shows the speed differential range between the two scenarios. Figure 7 includes additional graphs to show the speed differential between all three lanes.

Under scenario 2, speeds varied by 0.2 to $2.2 \mathrm{mph}$ (absolute values). Under scenario 3, speeds varied by 1.9 to $14.3 \mathrm{mph}$ (absolute values). A range of speed variations (e.g., 0.2 to $2.2 \mathrm{mph}$ in scenario (2) was due to the differences between segments and MPRs. Lowest speeds on dedicated
AV lanes was observed at segment 10 where the highest density, low traffic flow, and low average speeds were recorded as well.

Previous studies have suggested that high-speed differential, for example, between HOV lanes and General-Purpose Lanes (GPL) may warrant the installation of barriers between the two lanes $[30,37]$. The studies suggest that non-AV drivers may feel more comfortable driving with a maximum speed differential of no more than $15 \mathrm{mph}$ between lanes. They also suggest that speed differentials between 10 and $15 \mathrm{mph}$, while not warranting physical separation, may benefit from bufferseparated lanes such as double-line markings.

4.2.5. Key Microsimulation Findings. From a corridor capacity perspective, a level 3 automation AV-exclusive lane provides substantial benefits. Traffic flow was observed to increase by up to $14 \%$ depending on the corridor location and AV MPR. Similarly, density was observed to increase by up to $24 \%$. This can be attributed to the lower vehicle headways and more stable flows afforded by AVs. The additional capacity can be attributed to changes in AV driving dynamics and technology as opposed to an addition of a 
TABLE 3: Speed differential range (mph).

\begin{tabular}{lccccccc}
\hline \multirow{2}{*}{ Range } & Scenario 1 & AV 15\% & AV 30\% & AV 45\% & AVL 15\% & AVL 30\% & AVL 45\% \\
\hline Lower range & -0.7 & -1.9 & -2.2 & -1.4 & -6.0 & -9.4 & -14.3 \\
Upper range & 1.4 & 0.2 & 0.4 & 0.9 & -1.9 & -4.8 & -6.6 \\
\hline
\end{tabular}

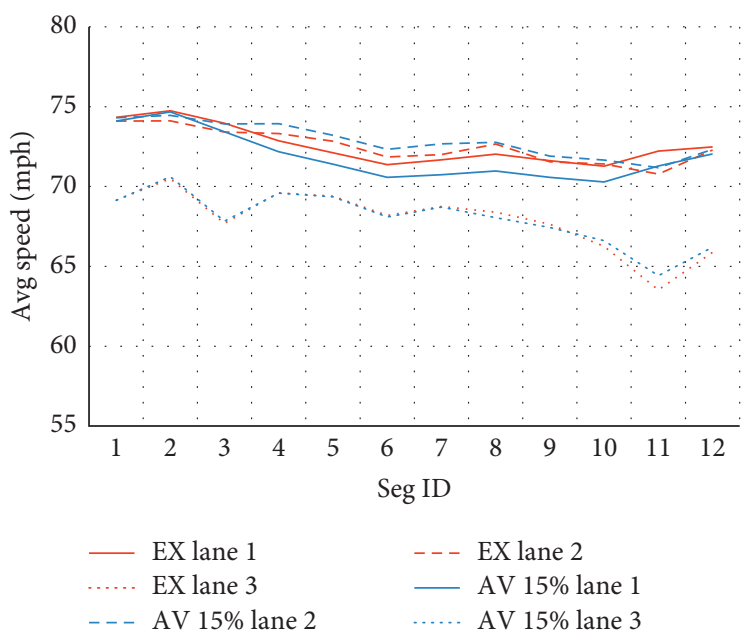

(a)

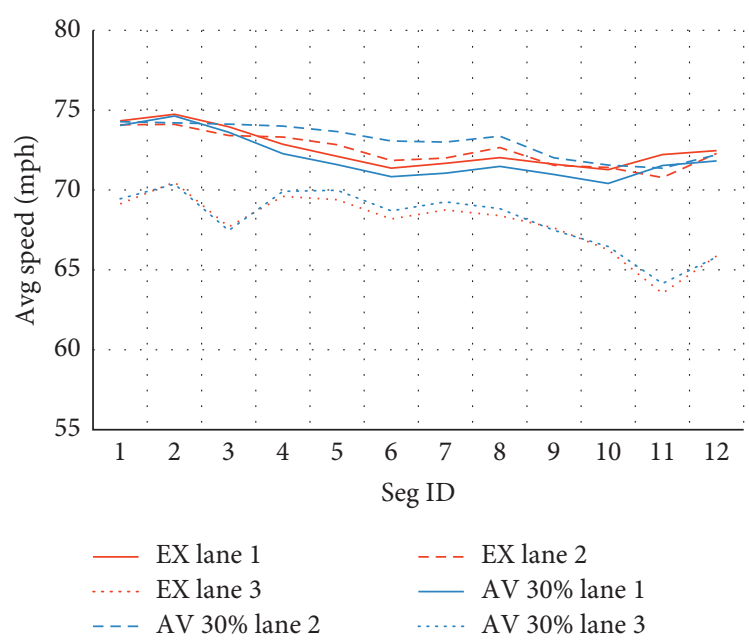

(c)

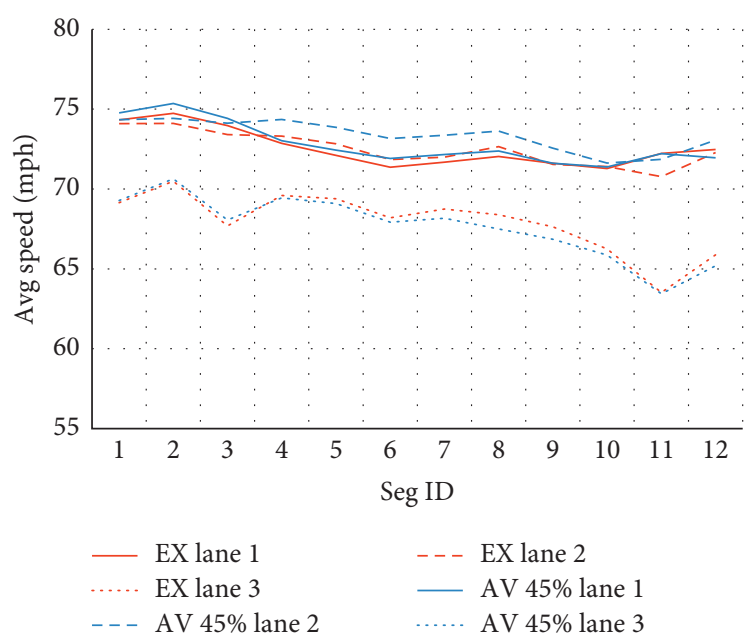

(e)

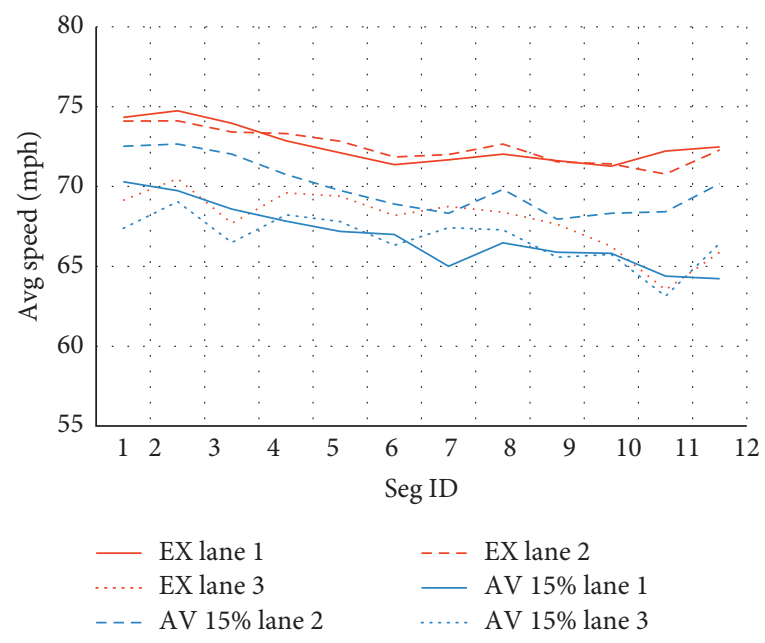

(b)

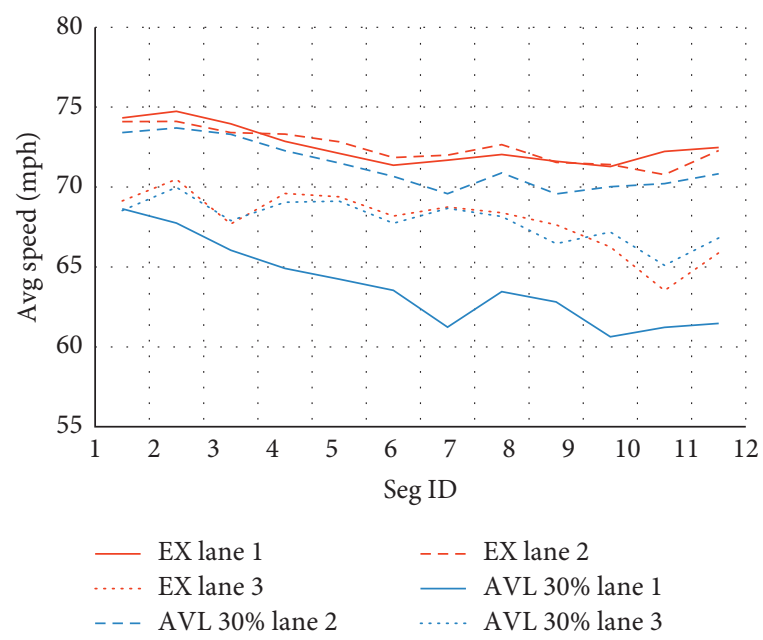

(d)

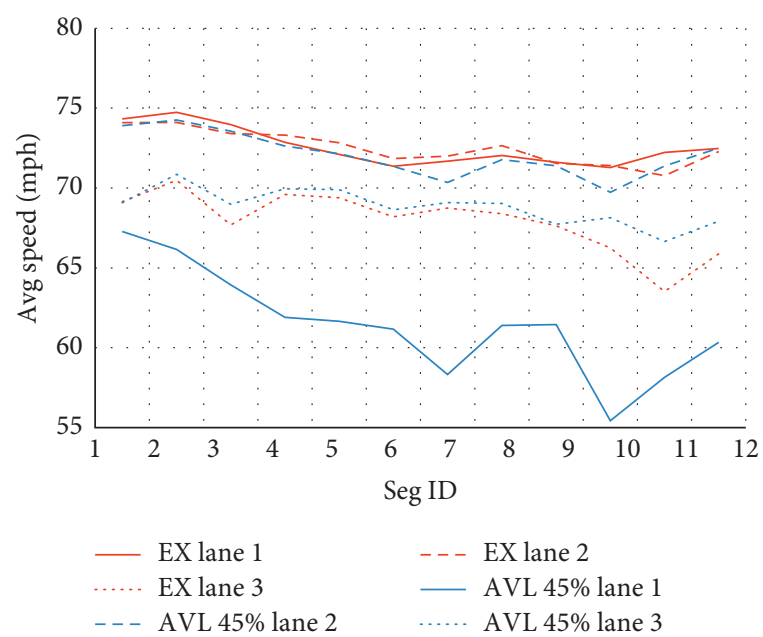

(f)

Figure 7: Speed differential between all three lanes. (a) Ex vs. AV 15\%, (b) Ex vs. AVL 15\%, (c) Ex vs. AV 30\%, (d) Ex vs. AVL 30\%, (e) Ex vs. AV 45\%, and (f) Ex vs. AVL 45\%. 
lane. It should be noted that the additional lane in scenario 3 was added to the northbound and did not impact the result of the southbound traffic analysis presented here.

From a safety perspective, an AV-exclusive lane does not identify any significant flaws and shows potential benefits. Despite capacity and density increasing, the average speed was observed to be 2 to $8 \mathrm{mph}$ lower depending on the location and AV MPR. In general, lower speeds can be attributed to lower crash severity.

This study does reveal the importance of understanding the impact of changing roadway characteristics, specifically the speed differential between the lanes 1 (adjacent to the left shoulder) and 2. The AV-exclusive lane introduces a distinction between lane characteristics that will require careful consideration if additional treatments or barriers are required.

Furthermore, AV vehicles may provide additional safety benefits not quantified in the microsimulation models. AVs have the potential to remove human error from the crash equation, the root cause of most accidents.

\section{Conclusions and Recommendations}

This study aims at evaluating the safety and operational impacts of a narrow AV-exclusive reversible lane on the existing I-15 expressway. This study identified significant risk factors contributing to crashes on I-15 and performed safety and operational impact analysis using traffic simulation. The following concluding remarks and recommendations are driven from the abovementioned tasks.

According to crash data analysis, unsafe speed is the most recurring primary collision factor (PCF) on I-15 ELs, the majority of which resulted in rear-end collisions. Implementation of an AV-exclusive lane could potentially reduce this type of crashes since AVs are expected to follow proper speed discipline with less variability and maintain sufficient bumper to bumper spacing. Improper turning and unsafe lane change are the next two most recurring PCF, the majority of which resulted in hit-object and sideswipe collisions. AVs' automated lateral control systems (e.g., LKA) could potentially reduce these collisions on an AVexclusive lane. However, highly reflective, clearly visible, and distinct lane markings, barriers, and signage are required for proper AV sensor operation. Also, the points of access from/ to the AV-exclusive lanes need to be carefully designed and monitored. The results of this study could be expanded in future research with statistical modelling approaches to identify the significant contributing factors when the crash data become available for AV-exclusive lanes.

Microsimulation findings indicate that an AV-exclusive lane may increase traffic flow and density by up to $14 \%$ and $24 \%$, respectively. This is achieved with lower vehicle headways and more stable flow afforded by AV-driving dynamics and technology. Microsimulation findings also indicate an AVexclusive lane has better speed limit compliance, and therefore, average speed is reduced. The lower speed may contribute to lower crash severity. However, the study reveals the importance of understanding the impact of roadway characteristics, specifically the speed differential between the exclusive lane and adjacent lane. An AV-exclusive lane introduces a distinction between lane characteristics that may result in an increase in speed differential which will require careful consideration if additional treatments or barriers are required.

Coinciding with San Diego County's dynamic change and growth, AVs will have an increasing presence on the area's transportation system. This study identified some of the leading causes of vehicular accidents on the I-15 express lanes, causes whose frequencies may be mitigated with the emergence of potentially safer handling AVs. With regional growth and corresponding traffic, AVs, which will have ever-increasing market penetration rates, may help with overall system performance as simulation demonstrated; under an exclusive lane scenario, the network achieved both reduction in speeds, as well as increase in throughput. The AV-exclusive lane provides an opportunity to safely and incrementally integrate AV technology into the greater transportation system, while, at the same time, realizing greater system performance.

\section{Data Availability}

The crash data used to support the findings of this study are publicly available at https://www.chp.ca.gov/ programs-services/services-information/switrs-internetstatewide-integrated-traffic-records-system.

\section{Conflicts of Interest}

The authors declare that there are no conflicts of interest regarding the publication of this paper.

\section{Acknowledgments}

This research was funded by the Safety through Disruption (Safe-D) National University Transportation Center (UTC), a grant from the U.S. Department of Transportation's University Transportation Centers Program (Federal Grant No. 69A3551747115). The contents of this paper reflect the views of the authors, who are responsible for the facts and the accuracy of the information presented herein.

\section{References}

[1] Critical Reasons for Crashes Investigated in the National Motor Vehicle Crash Causation Survey, National Highway Traffic Safety Administration, US Department of Transportation, Washington, DC, USA, 2018, https://crashstats.nhtsa.dot.gov/ Api/Public/ViewPublication/812506.

[2] A. Amditis, M. Bimpas, G. Thomaidis et al., "A situationadaptive lane-keeping support system: overview of the SAFELANE approach," IEEE Transactions on Intelligent Transportation Systems, vol. 11, no. 3, pp. 617-629, 2010.

[3] D. O. Cualain, M. Glavin, and E. Jones, "Multiple-camera lane departure warning system for the automotive environment," IET Intelligent Transport Systems, vol. 6, no. 3, p. 223, 2012.

[4] W.-W. Zhang, X.-L. Song, and G.-X. Zhang, "Real-time lane departure warning system based on principal component analysis of grayscale distribution and risk evaluation model," Journal of Central South University, vol. 21, no. 4, pp. 16331642, 2014. 
[5] P.-Y. Hsiao, C.-W. Yeh, S.-S. Huang, and L.-C. Fu, "A portable vision-based real-time lane departure warning system: day and night," IEEE Transactions on Vehicular Technology, vol. 58, no. 4, pp. 2089-2094, 2009.

[6] J. M. Clanton, D. M. Bevly, and A. S. Hodel, "A low-cost solution for an integrated multisensor lane departure warning system," IEEE Transactions on Intelligent Transportation Systems, vol. 10, no. 1, pp. 47-59, 2009.

[7] N. M. Enache, Y. Sebsadji, S. Mammar, B. Lusetti, and S. Glaser, "Driver's influence on the performance of an integrated lane departure avoidance and lane keeping assistance system," in Proceedings of the 2009 IEEE International Conference on Control Applications, pp. 119-124, Saint Petersburg, Russia, July 2009.

[8] Y. Wang, Y. Liu, H. Fujimoto, and Y. Hori, "Vision-based lateral state estimation for integrated control of automated vehicles considering multirate and unevenly delayed measurements," IEEE/ASME Transactions on Mechatronics, vol. 23, no. 6, pp. 2619-2627, 2018.

[9] J. Zhao, G. Lefranc, and A. El Kamel, "Lateral control of autonomous vehicles using multi-model and fuzzy approaches," IFAC Proceedings Volumes, vol. 43, no. 8, pp. 514-520, 2010.

[10] S. Mustaki, P. Chevrel, M. Yagoubi, and F. Fauvel, "Efficient multi-objective and multi-scenarios control synthesis methodology for designing a car lane centering assistance system," in Proceedings of the 2018 European Control Conference (ECC), pp. 929-934, Limassol, Cyprus, June 2018.

[11] S. Pendleton, H. Andersen, X. Du et al., "Perception, planning, control, and coordination for autonomous vehicles," $M a$ chines, vol. 5, no. 1, p. 6, 2017.

[12] R. Ismail, "Next-Generation Lane Centering Assist System: Design and Implementation of a Lane Centering Assist System, using NXP-BlueBox," Eindhoven University of Technology, Eindhoven, Netherlands, 2017, https://pure.tue. nl/ws/portalfiles/portal/91161739/2017_12_01_ASD_Ismail_ R.pdf.

[13] R. F. Berriel, E. De Aguiar, A. F. De Souza, and T. OliveiraSantos, "Ego-lane analysis system (ELAS): dataset and algorithms," Image and Vision Computing, vol. 68, pp. 64-75, 2017.

[14] A. Broggi, P. Medici, P. Zani, A. Coati, and M. Panciroli, "Autonomous vehicles control in the VisLab intercontinental autonomous challenge," Annual Reviews in Control, vol. 36, no. 1, pp. 161-171, 2012.

[15] J. Lee and B. Litkouhi, "A unified framework of the automated lane centering/changing control for motion smoothness adaptation," in Proceedings of the 2012 15th International IEEE Conference on Intelligent Transportation Systems, pp. 282-287, Anchorage, AK. USA, September 2012.

[16] A. Chebly, R. Talj, and A. Charara, "Coupled longitudinal and lateral control for an autonomous vehicle dynamics modeled using a robotics formalism," IFAC-PapersOnLine, vol. 50, no. 1, pp. 12526-12532, 2017.

[17] R. Attia, R. Orjuela, and M. Basset, "Combined longitudinal and lateral control for automated vehicle guidance," Vehicle System Dynamics, vol. 52, no. 2, pp. 261-279, 2014

[18] L. Xu, Y. Wang, H. Sun, J. Xin, and N. Zheng, "Integrated longitudinal and lateral control for Kuafu-II autonomous vehicle," IEEE Transactions on Intelligent Transportation Systems, vol. 17, no. 7, pp. 2032-2041, 2016.

[19] C. M. Filho, D. F. Wolf, V. Grassi, and F. S. Osorio, "Longitudinal and lateral control for autonomous ground vehicles," in Proceedings of the 2014 IEEE Intelligent Vehicles Symposium, pp. 588-593, Dearborn, MI, USA, June 2014.

[20] National Cooperative Highway Research Program and Transportation Research Board, and National Academies of Sciences, Engineering, and Medicine, Accident Modification Factors for Traffic Engineering and ITS Improvements, Transportation Research Board, Washington, DC, USA, 2008.

[21] F. Gross, P. P. Jovanis, K. Eccles, and K.-Y. Chen, Safety Evaluation of Lane and Shoulder Width Combinations on Rural, Two-Lane, Undivided Roads, Federal Highway Administration, Washington, DC, USA, 2009, https://rosap.ntl. bts.gov/view/dot/816.

[22] C. Lee, M. Abdel-Aty, J. Park, and J.-H. Wang, "Development of crash modification factors for changing lane width on roadway segments using generalized nonlinear models," Accident Analysis \& Prevention, vol. 76, pp. 83-91, 2015.

[23] S. Labi, S. Chen, P. V. Preckel, Y. Qiao, and W. Woldemariam, "Rural two-lane highway shoulder and lane width policy evaluation using multiobjective optimization," Transportmetrica A: Transport Science, vol. 13, no. 7, pp. 631-656, 2017.

[24] C. V. Zegeer, R. Stewart, F. Council, and T. R. Neuman, "Roadway widths for low-traffic-volume roads," NCHRP Report, no. 362, Washington, DC, USA, 1994, https://trid.trb. org/view/408237.

[25] F. Rosey, J.-M. Auberlet, O. Moisan, and G. Dupré, "Impact of narrower lane width," Transportation Research Record: Journal of the Transportation Research Board, vol. 2138, no. 1, pp. 112-119, 2009.

[26] P. B. W. Dorothy and S. L. Thieken, Trade-Off Considerations in Highway Geometric Design, Transportation Research Board, Washington, DC, USA, 2011.

[27] T. Giuffrè, A. Canale, A. Severino, and S. Trubia, "Automated vehicles: a review of road safety implications as a driver of change," in Proceedings of the 27th CARSP Conference, vol. 16, Toronto, Canada, June 2017.

[28] A. J. Turner and P. E Hick, "Inhibition of aldehyde reductase by acidic metabolites of the biogenic amines," Biochemical Pharmacology, vol. 24, no. 18, pp. 1731-1733, 1975.

[29] European Road Assessment Programme, Roads that Cars Can Read III: Tackling the Transition to Automated Vehicles, Road Safety Foundation, Basingstoke, UK, 2018.

[30] B. A. Hamilton, WSP, N. J. I. of Technology, National Cooperative Highway Research Program, and Transportation Research Board, and National Academies of Sciences, Engineering, and Medicine, Dedicating Lanes for Priority or Exclusive Use by Connected and Automated Vehicles, Transportation Research Board, Washington, DC, USA, 2018.

[31] L. Ye and T. Yamamoto, "Impact of dedicated lanes for connected and autonomous vehicle on traffic flow throughput," Physica A: Statistical Mechanics and its Applications, vol. 512, pp. 588-597, 2018.

[32] H. G. Seif and X. Hu, "Autonomous driving in the iCity-HD maps as a key challenge of the automotive industry," Engineering, vol. 2, no. 2, pp. 159-162, 2016.

[33] S. Bauer, Y. Alkhorshid, and G. Wanielik, "Using high-definition maps for precise urban vehicle localization," in Proceedings of the 2016 IEEE 19th International Conference on Intelligent Transportation Systems (ITSC), pp. 492-497, Rio De Janeiro, Brazil, November 2016.

[34] R. Liu, J. Wang, and B. Zhang, "High definition map for automated driving: overview and analysis," Journal of Navigation, vol. 73, no. 2, pp. 324-341, 2020. 
[35] S. Zheng and J. Wang, "High definition map-based vehicle localization for highly automated driving: geometric analysis," in Proceedings of the 2017 International Conference on Localization and GNSS (ICL-GNSS), pp. 1-8, Nottingham, UK, June 2017.

[36] W. Kühn, M. Müller, and T. Höppner, "Road data as prior knowledge for highly automated driving," Transportation Research Procedia, vol. 27, pp. 222-229, 2017.

[37] I. Hlavacek, M. Vitek, and R. Machemehl, Best Practices: Separation Devices between Toll Lanes and Free Lanes, Texas Department of Transportation, Austin, TX, USA, 2007, http://paper/ Best-Practices\%3A-Separation-Devices-between-Toll-andHlavacek-Vitek/86f3847f81f37e812b773178ee73891ce35802e8. 